\title{
OPTICS
}

\section{Multiphoton Ionization of Atoms and Ions by High-Intensity X-Ray Lasers}

\author{
S. B. Popruzhenko ${ }^{a, b}$, V. D. Mur ${ }^{a}$, V. S. Popov ${ }^{c}$, and D. Bauer ${ }^{b}$ \\ ${ }^{a}$ Moscow Institute of Engineering Physics, Kashirskoe sh. 31, Moscow, 115409 Russia \\ ${ }^{b}$ Max-Plank Institut für Kernphysik, Postfach 103980, 69029, Heidelberg, Germany \\ ${ }^{c}$ Institute for Theoretical and Experimental Physics, ul. Bol'shaya Cheremushkinskaya 25, Moscow, 117218 Russia \\ e-mail:poprz@theor.mephi.ru \\ Received December 31, 2008
}

\begin{abstract}
Coulomb corrections to the action function and rate of multiphoton ionization of atoms and ions in a strong linearly polarized electromagnetic field are calculated for high values of the Keldysh adiabaticity parameter. The Coulomb corrections significantly increase the ionization rate for atoms (by several orders of magnitude). An interpolation formula proposed for ionization rate is valid for arbitrary values of the adiabaticity parameter. The high accuracy of the formula is confirmed by comparison with the results of numerical calculations. The general case of elliptic polarization of laser radiation is also considered.
\end{abstract}

PACS numbers: 32.80. Rm, 32.80.Fb, 03.65.Sq

DOI: $10.1134 / \mathrm{S} 1063776109060053$

\section{INTRODUCTION}

Considerable advances have been made in recent years in the physics of the interaction of high-intensity laser fields with matter in connection with the development and commissioning of powerful sources of coherent radiation in the UV and X-ray wavelength ranges. Such sources are based on free-electron lasers. A unique device of this type (FLASH) operates at present at the DESY laboratory (Hamburg, Germany) $[1,2]$. In 2002, the FLASH device generated electromagnetic radiation pulses at a wavelength of about $100 \mathrm{~nm}$ (photon energy $\hbar \omega \approx 13 \mathrm{eV}$ ) with a duration on the order of $100 \mathrm{fs}$ and an intensity of up to $10^{13} \mathrm{~W} / \mathrm{cm}^{2}$ [3]. Since 2007, experiments with radiation pulses of a wavelength of $13 \mathrm{~nm}(\hbar \omega \approx 93 \mathrm{eV})$, a duration of about $10 \mathrm{fs}$, and an intensity of up to $10^{16} \mathrm{~W} / \mathrm{cm}^{2}$ have been carried out [4]. In the nearest future, transition to a wavelength of $6 \mathrm{~nm}$ is planned and use will be made of the third and fifth harmonics of the fundamental frequency (the latter harmonic corresponds to a wavelength of $1.2 \mathrm{~nm}$ ) [2]. Numerous experiments on the interaction of high-intensity coherent rf radiation with atoms, molecules, multiply charged ions, nanostructures, and solids were performed at the DESY laboratory in 2002-2007 using free-electron lasers (see [4-9] and the literature cited therein).

One of the fundamental nonlinear processes induced by a high-intensity laser field is multiphoton ionization of atoms, which was observed for the first time in 1965 [10] (on the modern state of the art, see reviews [11-14]). Until recently, the physics of interaction of high-intensity laser fields with matter has been mainly associated with experiments using highpower optical and IR lasers with a wavelength on the order of a micrometer or longer. Accordingly, theoretical investigations were also associated mainly with low frequencies of the laser field. It should be recalled that electromagnetic field in the theory of multiphoton ionization is assumed to be a low-frequency field if parameter $\gamma$, introduced by Keldysh [15], is much smaller than unity. Ionization in this case is known as tunnel-type ionization. The opposite limit $(\gamma \gg 1)$ is usually referred to as multiphoton ionization. When atoms and positive ions are ionized by the field of a high-intensity infrared optical laser, the Keldysh parameter is usually on the order of unity or smaller. The development of high-power X-ray lasers extends the experimental potential to the range of high frequencies and small wavelengths. Typical values of the Keldysh parameter in experiments [4, 7] amounted to $\gamma \approx 30-100$, which corresponds to the multiphoton ionization mode. Until recently, much less attention has been given to the development of the theory in this range of parameters. In this study, we analyze multiphoton ionization of atoms and positive ions in a strong high-frequency (rf) field (i.e., for $\gamma \gg 1$ ) and derive analytic expressions for the total ionization probability per unit time. The ionization rate calculated using these formulas is compared to the result of exact numerical calculations.

\footnotetext{
${ }^{1}$ Among powerful lasers, the most widely used are titanium-sapphire, neodymium, and $\mathrm{CO}_{2}$ lasers with wavelengths of $0.79-$ $0.80,1.06$, and $10.6 \mu \mathrm{m}$, respectively.
} 
In analyzing multiphoton ionization of atoms by high-intensity laser radiation, the Keldysh theory [15], also known as the strong field approximation [16], is widely used. In this approximation, the electromagnetic field of a wave is taken into account exactly, and the Coulomb interaction of an emitted electron with the atomic core is disregarded, which makes it possible to derive convenient analytic formulas for the ionization probability and momentum spectra of photoelectrons (see [17, 18] and the literature in [12]). For ionization of negative ions, the strong field approximation leads to good quantitative agreement with the results of exact numerical calculations and experimental data (see, for example, [19] and the literature therein). At the same time, in the case of neutral atoms and positive ions, the Coulomb interaction suppresses the potential barrier through which an electron tunnels, which considerably increases the ionization probability. For example, in a constant electric field of strength $\mathscr{E}$, ionization rate $w$ for the $s$ state of an atom differs from analogous quantity $w_{\mathrm{sr}}$ for an energy level in the short-range well [12, 20, 21] (with the same ionization potential $I$ ) by a factor of [22]

$$
\begin{gathered}
Q_{C}(\mathscr{E}, \omega=0) \equiv Q_{0}(\mathscr{E})=\frac{w(\mathscr{E})}{w_{\mathrm{sr}}(\mathscr{E})} \\
=\left(\frac{2 \hbar^{2} \kappa^{3}}{m e^{\mathscr{E}}}\right)^{2 n^{*}}=\left(\frac{2}{F}\right)^{2 n^{*}} \gg 1,
\end{gathered}
$$

where

$$
w_{\mathrm{sr}}(\mathscr{E})=C_{\kappa}^{2} \frac{\kappa^{2} \hbar}{m} \frac{\mathscr{E}}{\mathscr{E}} \exp \left(-\frac{2 \mathscr{E}_{\mathrm{ch}}}{3 \mathscr{E}}\right)
$$

Here, $k=\sqrt{2 m I} / \hbar$ and $\mathscr{E}_{\mathrm{ch}}=\hbar^{2} \kappa^{3} / m e$ are the characteristic momentum and the electric field of the atom (for the ground state of the hydrogen atom, $\mathscr{E}_{\mathrm{ch}} \equiv \mathscr{E}_{a}=m^{2} e^{5} / \hbar^{4}=$ $\left.5.14 \times 10^{9} \mathrm{~V} / \mathrm{cm}\right), n^{*}=\mathscr{L} e^{2} m / \hbar^{2} k=\mathscr{L}\left(I / I_{\mathrm{H}}\right)^{-1 / 2}$ is the effective principal quantum number of the level $\left(I_{\mathrm{H}}=\right.$ $m e^{4} / 2 \hbar^{2}=13.6 \mathrm{eV}$ is the ionization potential of the hydrogen atom), $m$ and $-e$ are the electron mass and charge, and $\mathscr{L}$ is the charge of the atomic core $(\mathscr{L}=1$ for neutral atoms and $\mathscr{L}=0$ for negative ions $\mathrm{H}^{-}, \mathrm{Na}^{-}$, etc.). Ratio $F=\mathscr{E} / \mathscr{E}_{\mathrm{ch}}$ is often referred to as the reduced field,

$$
F=0.169 \sqrt{\frac{\mathscr{g}}{1+\rho^{2}}}\left(\frac{I}{I_{\mathrm{H}}}\right)^{-3 / 2},
$$

where $\rho$ is the ellipticity of radiation $(-1 \leq \rho \leq 1 ; \rho=0$ and \pm 1 corresponds to the linear and circular polarizations of the wave, respectively), and $\mathscr{F}$ is the intensity measured in units of $10^{15} \mathrm{~W} / \mathrm{cm}^{2}$. Finally, $C_{\mathrm{K}}$ is the dimensionless asymptotic coefficient of the atomic wavefunction at distances $r \gg \hbar / \kappa$ from the nucleus.

\footnotetext{
${ }^{2}$ For the ground states of neutral atoms and singly charged ions, these coefficients are numerically close to unity [12]; for example, $C_{\mathrm{K}}=1,0.95$, and 1.04 for the $\mathrm{H}, \mathrm{K}$, and $\mathrm{Na}$ atoms, respectively, and $C_{\mathrm{K}}=1.15$ for the $\mathrm{H}^{-}$ion. A simple approximate formula for coefficients $C_{\mathrm{K}}$ was proposed by Hartree [23].
}

It should be emphasized that ionization potential $I$ and asymptotic coefficient $C_{\mathrm{K}}$ in expression (2) correspond to the atomic state for which the ionization probability is calculated rather than to the state in a short-range potential. Finally, the Keldysh parameter is defined as

$$
\gamma=\frac{\sqrt{2 m I} \omega}{e^{\mathscr{E}}}=\frac{1}{2 K_{0} F},
$$

where $K_{0}=I / \hbar \omega$ is the multiquantum parameter of the process. In the Keldysh theory, it was assumed that

$$
K_{0} \gg 1, \quad F \ll 1,
$$

here, parameter $\gamma$ can assume arbitrary values. Henceforth, we will use atomic units: $\hbar=e=m=1$.

Expression (1) for the Coulomb correction to the ionization rate is applicable not only in the case of a constant field, but also in the tunnel mode $(\gamma<1)$ for a plane electromagnetic wave with an arbitrary polarization. Usually, this correction increases the tunnel ionization probability by several orders of magnitude (this effect was reliably established in experiments; see, for example, [24]). At present, the expressions for tunnel ionization rate taking into account the Coulomb correction (and also known as the Ammosov-DeloneKrainov formulas [25]; see [26]) are widely used for calibrating the laser pulse intensity.

In this study, we obtain the Coulomb correction $Q_{C}(\mathscr{E}, \omega)$ in the opposite limit of high frequency, $\gamma \gg 1$, in the simplest case of ionization of the $s$ state of an atom or a positive ion by a linearly polarized field $(\rho=0)$. In this case, the formulas for tunnel ionization probability are completely inapplicable even for obtaining qualitative estimates. For example, for an intensity on the order of $10^{16} \mathrm{~V} / \mathrm{cm}^{2}$ used in experiment [4], successive tunnel ionization leads to the formation of ions up to $\mathrm{Xe}^{8+}-\mathrm{Xe}^{9+}$ instead of observed $\mathrm{Xe}^{21+}$ ions, and the tunnel ionization probability for the $\mathrm{Xe}^{10+}$ ion for the given intensity amounts to a value on the order of $10^{-24}$ for a pulse with a duration of about $10 \mathrm{fs}$. In our calculations, we use the imaginary time method (ITM), in which subbarrier trajectories satisfying the classical equation of motion, but in imaginary time, are considered for describing quantum tunneling through a barrier $[27,28]$. In the derivation of the Coulomb correction, we will use the Kapitza method $[29,30]$ for describing the averaged motion of a particle in a rapidly oscillating field. The application of the ITM for analyzing Coulomb effects in the spectral-angular distributions of photoelectrons in a high-intensity laser field is considered in detail in [31].

The article has the following structure. In Section 2, the method for calculating the Coulomb correction in a linearly polarized rf field is formulated and an explicit expression is derived for this correction. In Section 3 , the results are analyzed and compared with 
the results of numerical calculations. In Section 4, the general case of elliptically polarized field is considered. In Section 5, the applicability conditions for the derived analytic formulas are formulated. Appendices contain the description of the Kapitza averaging method applied to the photoionization problem, details of calculations for a linearly polarized field, and a brief description of numerical methods used for controlling the accuracy of the analytic formula derived here. Preliminary results of this study were announced in [32].

\section{COULOMB CORRECTION IN A LINEARLY POLARIZED FIELD}

\subsection{Imaginary Time Method in the Ionization Problem}

The probability of ionization of a level with a binding energy of $-I$ by an electromagnetic field with amplitude $\mathscr{E}_{0}$ and characteristic frequency $\omega$ in the ITM is controlled (with an exponential error) by the imaginary part of a function of truncated action calculated along the trajectory on which an electron has a preset momentum $p[18,28]$ :

$$
w\left(\mathscr{E}_{0}, \omega, \mathbf{p}\right) \propto \exp \left[-2 \operatorname{Im} W\left(\mathscr{E}_{0}, \omega, \mathbf{p}\right)\right] .
$$

For a particle bound by short-range forces, the action and the trajectory are controlled by the field of the electromagnetic wave. In a wave with vector potential $A(t)$ and electric field $\mathscr{E}(t)=-\dot{\mathbf{A}}(t) / c$ ( $c$ is the velocity of light), truncated action $W_{0}$ and Lagrange function $\mathscr{L}_{0}$ have the form ${ }^{3}$

$$
\begin{gathered}
W_{0}=\int_{t_{s}(\mathbf{p})}^{\infty}\left(\mathscr{L}_{0}-I\right) d t+F_{0}(\mathbf{p}), \\
\mathscr{L}_{0}=\frac{1}{2} \mathbf{v}_{0}^{2}-\mathscr{E}(t) \mathbf{r}_{0}, \\
F_{0}(\mathbf{p})=-\left.\left(\mathbf{v}_{0} \cdot \mathbf{r}_{0}\right)\right|_{t=t_{s}} ^{t \rightarrow \infty}=\mathbf{p} \cdot\left[\mathbf{p} t_{s}+\mathbf{G}\left(t_{s}\right)\right],
\end{gathered}
$$

where

$$
\mathbf{G}(t)=\frac{1}{c} \int^{t} d t^{\prime} \mathbf{A}\left(t^{\prime}\right)
$$

and the value of the function at point $t \longrightarrow \infty$, with a purely real contribution, which does not affect the probability, is omitted on the right-hand side of the expression for $F_{0}(\mathbf{p})$. Subscripts " 0 " indicate that all quantities are evaluated taking into account the laser field alone; i.e., the trajectory is a solution to the Newton equation disregarding the Coulomb interaction,

$$
\ddot{\mathbf{r}}_{0}=\dot{\mathbf{v}}_{0}=-\mathscr{E}(t) \text {. }
$$

\footnotetext{
${ }^{3}$ These formulas are one of the main results of the ITM in the theory of ionization of atoms by an ac electric field. The derivation of these formulas can be found in $[27,28]$.
}

The trajectory begins at a complex instant $t_{s}(\mathbf{p})$ that can be determined from the equation

$$
\left(\mathbf{p}+\frac{1}{c} \mathbf{A}\left(t_{s}\right)\right)^{2}+\kappa^{2}=0 .
$$

The same equation also defines the initial complex velocity $\mathbf{v}_{0}\left(t_{s}\right)=\mathbf{p}+\mathbf{A}\left(t_{s}\right) / c$. The trajectory itself has the form

$$
\begin{gathered}
\mathbf{v}_{0}(t)=\mathbf{p}+\frac{1}{c} \mathbf{A}(t), \\
\mathbf{r}_{0}(t)=\mathbf{X}_{0}+\mathbf{p}\left(t-t_{s}\right)+\mathbf{G}(t)-\mathbf{G}\left(t_{s}\right)
\end{gathered}
$$

Quantity $\mathbf{X}_{0}$ has the meaning of the initial point of subbarrier motion, $\mathbf{X}_{0}(\mathbf{p})=\mathbf{r}_{0}\left(t=t_{s}(\mathbf{p})\right)$. Usually, we set $\mathbf{X}_{0}=0$; in this case, the trajectory in real time for a nonzero value of momentum $\mathbf{p}$ has a constant imaginary part [28].

To calculate the total ionization probability with an exponential accuracy, it is sufficient to find action (7) along the trajectory corresponding to the most probable value of finite momentum $\mathbf{p}_{0}$. We will henceforth consider a field linearly polarized along the $x$ axis,

$$
\mathscr{E}(t)=-\mathscr{E}_{0} \cos \omega t
$$

in this case ${ }^{4}, \mathbf{p}_{0}=0$. The corresponding trajectory is one-dimensional and directed along the polarization vector:

$$
\begin{gathered}
x_{0}(\tau)=\frac{\mathscr{E}_{0}}{\omega^{2}}\left(\cosh \tau_{0}-\cosh \tau\right), \\
\tau_{0} \geq \tau \equiv-i \omega t \geq 0, \\
x_{0}(\theta)=\frac{\mathscr{E}_{0}}{\omega^{2}}\left(\cosh \tau_{0}-\cos \theta\right), \quad \theta \equiv \omega t \geq 0,
\end{gathered}
$$

while the stationary point corresponding to it has the form $^{5}$

$$
\begin{gathered}
\omega t_{s}\left(\mathbf{p}_{0}=0\right)=i \tau_{0}, \\
\tau_{0}=\operatorname{arcsinh} \gamma \approx\left\{\begin{array}{l}
\gamma, \gamma \ll 1, \\
\ln (2 \gamma), \quad \gamma \gg 1 .
\end{array}\right.
\end{gathered}
$$

\section{Quantity}

\footnotetext{
4 To be more precise, the most probable energy of a photoelectron is not necessarily zero, but it does not exceed the photon energy so that $0 \leq p_{0}<\sqrt{2 \omega}$ and $p_{0} / \kappa<1 / \sqrt{K_{0}} \ll 1$.

${ }^{5}$ Here, we have chosen the trajectory along which the electron moves to the right (in the positive direction of the $x$ axis) so that $\mathscr{E}\left(\tau_{s}\right)=-\mathscr{E}_{0} \cosh \tau_{0}<0$. A symmetric trajectory passing to the left of the atom begins at the instant $\omega t_{s}=\pi+i \tau_{0}$ and makes the same contribution to the ionization probability.
} 


$$
\begin{gathered}
b \equiv x_{0}(\theta=0)=\frac{\mathscr{E}_{0}}{\omega^{2}}\left(\sqrt{1+\gamma^{2}}-1\right) \\
\approx\left\{\begin{array}{l}
\left(I / \mathscr{E}_{0}\right)\left(1-\gamma^{2} / 4\right), \quad \gamma \ll 1, \\
(\sqrt{2 I} / \omega)\left(1-\gamma^{-1}\right), \quad \gamma \gg 1
\end{array}\right.
\end{gathered}
$$

has the meaning of the tunnel barrier width. An electron begins moving in real time at this point. It should be noted that if conditions (5) hold, the barrier width is always much larger than the size of the bound state, $\kappa b \gg 1$, which ensures the applicability of the semiclassical approximation (ITM in our case).

\subsection{Coulomb Corrections}

The Coulomb interaction between an emitted electron and the atomic core can be taken into account assuming that the trajectories and action introduced above are the zeroth approximation and calculating corrections to these quantities [22]. Naturally, this method is applicable when the Coulomb field is small in a certain sense as compared to the laser field. The smallness criterion will be formulated below (see also $[31,33])$. In this case, two types of corrections appear. First, Coulomb interaction energy $U_{C}=-\mathscr{L} / r$ leads to the following correction to action:

$$
W_{C 0}=-\int_{t_{s}}^{\infty} U_{C}\left(\mathbf{r}_{0}(t)\right) d t=\mathscr{L} \int_{t_{s}}^{\infty} \frac{d t}{\sqrt{\mathbf{r}_{0}^{2}(t)}} .
$$

Since this correction explicitly contains a "small" Coulomb interaction (it is proportional to charge $\mathscr{E}$ ), the integral can be evaluated along a trajectory unperturbed by the Coulomb field. At the lower limit, the integral diverges logarithmically and requires matching to the asymptotic form of the wavefunction of a free atom. The matching procedure is described in detail in [22, 31, 33] (see Section 4 below, in which correction (15) is calculated in the case of elliptic polarization). For a linearly polarized field, such a calculation leads to familiar result (1). It turns out that correction (1) has the same form in the tunnel and multiphoton limits and is formally independent of frequency altogether; however, this applies only to fields with linear polarization. ${ }^{6}$ This correction always increases the ionization probability. In the tunnel mode, the effect of increasing ionization probability can easily be interpreted since the Coulomb field lowers the barrier through which an electron tunnels. The fact that expression (1) for the Coulomb correction is "pulled" into the multiphoton region was established for the first time in [22].

\footnotetext{
${ }^{6}$ This statement is correct for a strictly monochromatic field with a constant temporal envelope. For short pulses, the correction is a function of frequency even in the case of linear polarization [33]; see also Appendix D.
}

Another correction appears due to distortion of the electron trajectory by the Coulomb field. If correction $\mathbf{r}_{1}(t)$ to the trajectory has been determined in a particular way (e.g., numerically), the corresponding correction to the action is

$$
W_{C 1}=\int_{t_{s}}^{\infty}\left[\mathbf{v}_{0} \cdot \mathbf{v}_{1}+\frac{1}{2} \mathbf{v}_{1}^{2}-\mathscr{E}(t) \mathbf{r}_{1}\right] d t+F_{1}
$$

where

$$
F_{1}=-\left.\left(\mathbf{v}_{0} \cdot \mathbf{r}_{1}+\mathbf{v}_{1} \cdot \mathbf{r}_{0}+\mathbf{v}_{1} \cdot \mathbf{r}_{1}\right)\right|_{t=t_{s}} ^{t \rightarrow \infty}
$$

To calculate correction (16), we first consider the part of the trajectory behind the barrier for which time is real. For $\gamma \gg 1$, we can average over fast oscillations in the laser field and consider only the smooth motion in the Coulomb field [33]. In this approximation, the problem becomes conservative, and the energy conservation law gives the relation between momentum $p_{\|}$ at the exit from under the barrier (for the $1 \mathrm{D}$ trajectory considered here, the momentum is directed along the polarization vector of the wave) and momentum $p_{0}=0$ at infinity. Details of calculations are given in Appen$\operatorname{dix} \mathrm{A}$, and the results have the form

$$
p_{\|}=\sqrt{p_{0}^{2}+2 \mathscr{L} / b}=\sqrt{2 n^{*} \omega}=\kappa \sqrt{2 \mu} .
$$

This formula contains a small parameter

$$
\mu=\frac{\mathscr{L} \omega}{\kappa^{3}}=\frac{n^{*}}{2 K_{0}} \ll 1,
$$

determining the contribution of the Coulomb field to the distortion of the electron trajectory.

In imaginary time (i.e., for $0<\tau \leq \tau_{0}$ ), the Coulomb correction to trajectory (12) can be determined from the equation

$$
\ddot{x}_{1}=-\frac{\mathscr{Z}}{x_{0}^{2}}
$$

The solution to this equation corresponding to momentum (18) at instant $\omega t=0$ is obtained in Appendix A (see also [33]):

$$
\begin{gathered}
x_{0}(\tau)=b(1-u), \\
x_{1}(\tau)=b(a+i \sqrt{2 \mu} \tau \\
\left.+\mu\left[\frac{1}{2} \tau^{2}-\ln (1-u)+L_{2}(u)\right]\right), \\
u=\exp \left(\tau-\tau_{0}\right),
\end{gathered}
$$

where $L_{2}$ is the Euler logarithm [34], 


$$
\begin{gathered}
L_{2}(u)=-\int_{0}^{u} \frac{\ln (1-t)}{t} d t=\sum_{k=1}^{\infty} \frac{u^{k}}{k^{2}} \\
u+\frac{1}{4} u^{2}+O\left(u^{3}\right), \quad u \longrightarrow 0, \\
\frac{\pi^{2}}{6}+(1-u)[\ln (1-u)-1]+\ldots, \quad u \rightarrow 1 .
\end{gathered}
$$

It can be seen that the correction to the trajectory in imaginary time is complex-valued. Real-valued constant $a$ vanishes for $\mu \longrightarrow 0$ and does not change the result.

The contribution from the correction to trajectory (21) to action (16) is calculated in Appendix B. The imaginary part of the action is given by

$$
\operatorname{Im} W_{C 1}=\mu \frac{\kappa^{2}}{\omega} \tau_{0} .
$$

Substitution of this quantity into the exponent in relation (6) leads to the emergence of an additional factor

$$
Q_{1}=\exp \left(-2 \operatorname{Im} W_{C 1}\right)=(2 \gamma)^{-2 n^{*}}, \quad \gamma \gg 1 .
$$

in the ionization probability. Simple, although not rigorous, analysis proves the correctness of asymptotic form (23) for $Q_{1}$ and even makes it possible to refine it.

In accordance with relation (18), $p_{\|} \geq \kappa \sqrt{2 \mu}$ at the instant when the electron emerges from under the barrier (the lower boundary corresponds to the photoionization threshold). Such a momentum is required for the electron to overcome the Coulomb attraction during its motion above the barrier averaged over fast oscillations. On the other hand, the probability of extraction of the electron from the potential well (short-range potential) decreases upon an increase in longitudinal momentum $p_{\|}[12,18]$ :

$$
\begin{gathered}
w_{\mathrm{sr}}\left(p_{\|}\right)=w_{\mathrm{sr}}(0) \exp \left\{-c_{1}(\gamma) \frac{p_{\|}^{2}}{\omega}\right\}, \\
c_{1}(\gamma)=\operatorname{arcsinh} \gamma-\frac{\gamma}{\sqrt{1+\gamma^{2}}} .
\end{gathered}
$$

Substituting $p_{\|}^{2}=2 \mu \kappa^{2}$ and $\mu \kappa^{2} / \omega=n^{*}$ into this expression and considering that $c_{1}(\gamma)=\ln (2 \gamma)-1+$ $O\left(\gamma^{-2}\right)$ for $\gamma \gg 1$, we immediately arrive at relation (23).

Thus, the total Coulomb correction can be written in the form

$$
Q_{C}\left(\mathscr{E}_{0}, \omega\right)=Q_{0} Q_{1}=Q_{0} \exp \left\{-2 n^{*} c_{1}(\gamma)\right\},
$$

which is valid for arbitrary values of adiabaticity parameter $\gamma$.

It should be noted that the imaginary part of function $F_{1}(17)$ was not taken into account in [33], which led to an erroneous result. Formula (25) derived above and having the same meaning as formulas (14) and (20) in [33] corrects this inaccuracy.

\section{DISCUSSION OF RESULTS AND COMPARISON WITH NUMERICAL CALCULATIONS}

Coulomb correction (25) contains two factors, one of which, $Q_{0}$, is independent of the adiabaticity parameter and the other, $Q_{1} \longrightarrow 0$, tends to unity for $\gamma \ll 1$ and $Q_{1} \ll 1$ for $\gamma \gg 1$ as follows from (23). Thus, Coulomb correction $Q_{1}$ obtained here effectively lowers the ionization probability in the multiphoton region; however, total correction (25) remains numerically large:

$$
Q_{C} \approx\left(2 e K_{0}\right)^{2 n^{*}} \gg 1, \quad \gamma \gg 1 .
$$

The ionization rate for the $s$ state in the short-range potential is given by $[12,17,18]$

$$
\begin{aligned}
w_{\mathrm{sr}}= & \frac{\kappa^{2}}{\pi} C_{\kappa}^{2} K_{0}^{-3 / 2} \beta^{1 / 2} \sum_{N \geq N_{\mathrm{th}}} \mathscr{F}\left(\sqrt{\beta\left(N-N_{\mathrm{th}}\right)}\right) \\
& \times \exp \left\{-\frac{2 g(\gamma)}{3 F}-2 c_{1}\left(N-N_{\mathrm{th}}\right)\right\}
\end{aligned}
$$

where

$$
\begin{gathered}
g(\gamma)=\frac{3}{2 \gamma}\left[\left(1+\frac{1}{2 \gamma^{2}}\right) \operatorname{acrsinh} \gamma-\frac{\sqrt{1+\gamma^{2}}}{2 \gamma}\right], \\
\beta=\frac{2 \gamma}{\sqrt{1+\gamma^{2}}}, \\
\mathscr{F}(x)=\int_{0}^{x} d y \exp \left(-x^{2}+y^{2}\right)=x_{1} F_{1}\left(1,3 / 2 ;-x^{2}\right)
\end{gathered}
$$

is the Dawson function [35] and $N_{\text {th }}=K_{0}\left(1+1 / 2 \gamma^{2}\right)$ is the threshold number of quanta required for ionization of the level.

The ionization rate of the $s$ state is given by ${ }^{7}$

$$
w=Q_{C} w_{\mathrm{sr}} \text {. }
$$

In the low-frequency limit $\gamma \ll 1$, correction $Q_{1} \longrightarrow 1$, and expression (28) is transformed into the familiar formula for the tunnel ionization rate for the $s$ level in a low-frequency field [22, 25],

$$
w=2 C_{\kappa}^{2} \kappa^{2} \sqrt{\frac{3 F}{\pi}}\left(\frac{2}{F}\right)^{2 n^{*}-1} \exp \left(-\frac{2}{3 F}\right),
$$

which differs from relations (1) and (2) by a factor of $\sqrt{3 F / \pi}$. This additional factor [18] is connected with the time variation of the field (it should be recalled that ionization rate (2) corresponds to a static field).

Consequently, although result (25) was obtained for $\gamma \gg 1$, it ensures the correct static limit $\gamma \longrightarrow 0$. In

\footnotetext{
${ }^{7}$ It should be noted that the ionization rate for an atom in [36] was also calculated using formula (28); probability $w_{\text {sr }}$ was determined numerically for arbitrary values of parameter $\gamma$, but the Coulomb correction was calculated in tunnel limit (1).
} 

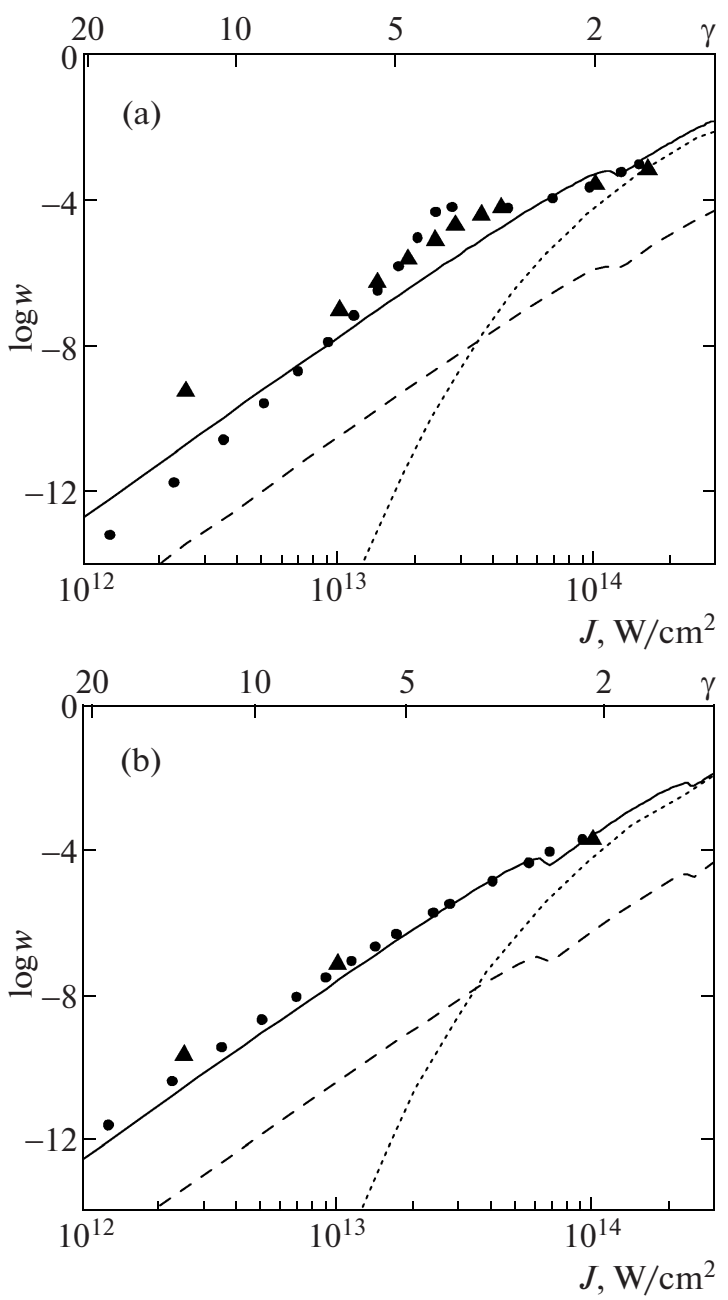

Fig. 1. Ionization rate (in atomic units; 1 a.u. $=4.13 \times$ $10^{16} \mathrm{~s}^{-1}$ ) calculated by formulas (28) (solid curve), (27) (dashed curve), and tunnel formula (29) (dotted curve), as well as determined numerically by the Floquet method $(\bullet)$ and from the solution of the time-dependent Schrödinger equation $(\mathbf{\Lambda})$ as a function of intensity for the $1 s$ state of the hydrogen atom in the field of a linearly polarized wave of lengths $\lambda=400 \mathrm{~nm}$ (a) and $422 \mathrm{~nm}$ (b). Parameters $K_{0}$ are 4.39 and 4.63 , respectively. Intensities of $10^{14}$ and $10^{13} \mathrm{~W} / \mathrm{cm}^{2}$ corresponds to field amplitudes $\mathscr{E}_{0}=$ $0.053 \mathscr{E}_{a}$ and $0.0169 \mathscr{E}_{a}$. The Coulomb correction is $Q_{C} \approx$ $6 \times 10^{2}$.

an intermediate range of $\gamma \approx 1$, formula (25) for the Coulomb correction can be treated as an interpolation formula. Since quantity $Q_{C}$ is a monotonic function of parameters, the accuracy of such an interpolation turns out to be quite high, which is confirmed by comparison with the results of numerical calculations given below. Thus, expressions (25), (27), and (28) define the ionization probability of the $s$ states of atoms and positive ions for arbitrary values of the adiabaticity parameter (provided that ionization is of the multiphoton type; i.e., $K_{0} \gg 1$ ). It should also be noted that $\exp \left[-2 n^{*} c_{1}(\gamma)\right] \approx\left(1+2 e^{-1} \gamma\right)^{-2 n^{*}}$ to a high degree of accuracy, which allows us to represent correction
(25) in a simpler form convenient for obtaining estimates:

$$
Q_{C} \approx\left(\frac{2}{F}\right)^{2 n^{*}}\left(1+2 e^{-1} \gamma\right)^{-2 n^{*}}, \quad e=2.718 \ldots
$$

In the limit $\gamma \gg 1$, correction (26) is independent of intensity and is numerically large. Far from the closure of multiphoton ionization channels, ${ }^{8}$ the Dawson function in (27) is on the order of unity and the largest contribution to the sum comes from the first term; i.e., ionization occurs with absorption of the minimal possible number of photons, $N_{\min }$. The expression for ionization rate in this case assumes the following simple form:

$$
\begin{gathered}
w \approx \frac{I}{\hbar} A\left(K_{0}, n^{*}\right) F^{2 N_{\min }}, \\
A\left(K_{0}, n^{*}\right) \approx 2^{2 n^{*}} C_{\mathrm{\kappa}}^{2} e^{N_{\min }+2 n^{*}} K_{0}^{2 N_{\min }+2 n^{*}-3 / 2} .
\end{gathered}
$$

Thus, a power law typical of perturbation theory of $\operatorname{order} N_{\text {min }}$ is reproduced, which is natural in the multiphoton mode $[12,15]$. It is important, however, that we have also obtained an explicit value of coefficient $A\left(K_{0}, n^{*}\right)$, which is in good agreement with the results of numerical calculations (curves in Fig. 1). This coefficient is numerically large and is the product of large numbers; consequently, it is quite sensitive to even insignificant changes in parameters. Coulomb correction (25) makes a significant contribution to the value of $A$. For parameters $K_{0} \approx 5-10$ and $n^{*} \approx 1-3$ typical of ionization of atoms and ions in a strong field, the value of $Q_{C}$ varies in the interval $10^{2}-10^{10}$. Factor $Q_{1}$ responsible for the deviation from the tunnel limit is also of fundamental importance: $Q_{1} \approx 10^{-9}-10^{-1}$.

It should be noted that a simple analytic expression for the ionization rate of atoms in the multiphoton mode was derived by Bersons in [37] for $n^{*} \geq N_{\min }$. For $K_{0} \gg 1$, this condition indicates that we are dealing with ionization of the upper-lying Rydberg level and that parameter (19) introduced above is large, $\mu>1$. Our results are applicable in the opposite limit $\mu<1$, $K_{0} \gg n^{*}$ and, hence, do not ensure the limiting transition to one-photon ionization, $N_{\min }=1$. Conversely, the Bersons formula for $N_{\min }=1$ is transformed into the well-known Kramers relation for the photoeffect [38]. In addition, the ionization rate determined in [37] is averaged over the orbital quantum number $l$ of the Rydberg level, while our result corresponds to $l=0$. As a consequence, the expression derived in [37] for a coefficient analogous to $A\left(K_{0}, n^{*}\right)$ differs substantially from relation (31).

Tables 1 and 2 illustrate the effect of Coulomb corrections on the ionization rate of atoms and ions in the field of IR and X-ray lasers.

\footnotetext{
${ }^{8}$ The closure of a channel is a change in the minimal number of photons required for ionization, $N_{\min }=\left[K_{0}\right]+1$, by unity $\left(\left[K_{0}\right]\right.$ is the integral part of number $K_{0}$ ); i.e., $N_{\text {th }}=N_{\text {min }}$.
} 
Table 1. Coulomb corrections for neutral atoms

\begin{tabular}{l|c|c|c|c|c|c|c|c|c|l}
\hline \multicolumn{1}{c|}{ Atom } & $I, \mathrm{eV}$ & $F$ & $K_{0}$ & $n^{*}$ & $\mu$ & $\gamma$ & $Q_{C}$ & $Q_{1}$ & $w_{\text {sr }}$ & $w$ \\
\hline $\mathrm{K}$ & 4.34 & 0.094 & 2.77 & 1.78 & 0.321 & 1.90 & $8.2(+3)$ & 0.16 & $3.0(-5)$ & 0.25 \\
$\mathrm{Na}$ & 5.14 & 0.073 & 3.29 & 1.63 & 0.248 & 2.06 & $7.5(+3)$ & 0.15 & $1.1(-5)$ & $8.3(-2)$ \\
$\mathrm{Li}$ & 5.39 & 0.068 & 3.48 & 1.59 & 0.228 & 2.12 & $7.1(+3)$ & 0.15 & $6.4(-6)$ & $4.6(-2)$ \\
$\mathrm{I}$ & 10.5 & 0.025 & 6.77 & 1.14 & 0.084 & 2.96 & $3.0(+3)$ & 0.14 & $2.0(-11)$ & $6.1(-8)$ \\
$\mathrm{Xe}$ & 12.1 & 0.020 & 7.81 & 1.06 & 0.068 & 3.18 & $2.4(+3)$ & 0.14 & $3.9(-13)$ & $9.4(-10)$ \\
$\mathrm{H}$ & 13.6 & 0.017 & 8.77 & 1.00 & 0.057 & 3.37 & $2.0(+3)$ & 0.14 & $7.5(-15)$ & $1.5(-11)$ \\
$\mathrm{He}$ & 24.6 & 0.007 & 15.8 & 0.74 & 0.023 & 4.53 & $7.2(+2)$ & 0.16 & $5.1(-28)$ & $3.7(-25)$ \\
\hline
\end{tabular}

Note: The table contains parameters $F, K_{0}, \mathrm{n}^{*}, \mu$, and $\gamma$, as well as Coulomb correction (25), correction $Q_{1}$, ionization rate (27) from the short-range potential with the same value of binding energy as in the corresponding atom, and analogous quantity (28) taking into account the Coulomb correction for neutral atoms in the field of a titanium-sapphire laser $(\hbar \omega \approx 1.55 \mathrm{eV}, \lambda \approx 800 \mathrm{~nm})$ for intensity $J=10^{13} \mathrm{~W} / \mathrm{cm}^{2}$. Coulomb correction $Q_{1}$ was taken in the form $Q_{1}(\gamma)=\exp \left[-2 n^{*} c_{1}(\gamma)\right]$. Notation $a(b) \equiv a \times 10^{b}$ is used. The ionization probability during the time of action of the pulse can be obtained by multiplying the reduced ionization rate by the pulse duration in atomic units: $100 \mathrm{fs} \approx 2.5 \times 10^{3} \mathrm{a}$.u. In the case of $\mathrm{K}, \mathrm{Na}$, and $\mathrm{Li}$ atoms for which the value of this quantity is greater than unity, saturation of single ionization occurs before termination of the pulse.

Table 2. Coulomb corrections for positive ions

\begin{tabular}{l|c|l|l|l|l|l|l|l|l|l}
\hline \multicolumn{1}{c}{ Ion } & $I, \mathrm{eV}$ & $F$ & $K_{0}$ & $n^{*}$ & $\mu$ & $\gamma$ & $Q_{C}$ & $Q_{1}$ & $w_{\text {sr }}$ & \multicolumn{1}{c}{$w$} \\
\hline $\mathrm{Xe}^{2+}$ & 32 & 0.015 & 2.52 & 1.96 & 0.388 & 13 & $2.7(+4)$ & $1.3(-4)$ & $1.2(-8)$ & $3.5(-4)$ \\
$\mathrm{Xe}^{3+}$ & 47 & 0.0083 & 3.70 & 2.15 & 0.291 & 16 & $4.0(+5)$ & $2.3(-5)$ & $6.2(-12)$ & $2.5(-6)$ \\
$\mathrm{Xe}^{4+}$ & 60 & 0.0057 & 4.72 & 2.38 & 0.252 & 18 & $5.1(+6)$ & $4.1(-6)$ & $3.5(-15)$ & $1.8(-8)$ \\
$\mathrm{Xe}^{5+}$ & 72 & 0.0044 & 5.67 & 2.61 & 0.230 & 20 & $5.8(+7)$ & $7.8(-7)$ & $1.9(-18)$ & $1.1(-10)$ \\
$\mathrm{Ar}^{2+}$ & 41 & 0.010 & 3.23 & 1.73 & 0.268 & 15 & $2.0(+4)$ & $2.4(-4)$ & $2.1(-11)$ & $4.1(-7)$ \\
$\mathrm{Ar}^{3+}$ & 60 & 0.0058 & 4.72 & 1.91 & 0.202 & 18 & $2.3(+5)$ & $4.9(-5)$ & $4.5(-15)$ & $1.1(-9)$ \\
\hline
\end{tabular}

Note: The same as in Table 1 for positive ions in the field of an X-ray laser with a photon energy $\hbar \omega \approx 12.7 \mathrm{eV}(\lambda \approx 98 \mathrm{~nm})$ and an intensity of $10^{14} \mathrm{~W} / \mathrm{cm}^{2}$.

It can be seen from Table 1 that in the case of ionization of atoms by the field of a titanium-sapphire laser, the adiabaticity parameter is not very large $(\gamma \approx$ $2-3)$ for reasonable values of intensity. Accordingly, the deviation of the Coulomb correction from its tunnel limit (1), characterized by quantity $Q_{1}$, is insignificant ( $Q_{1} \approx 0.1$; see Fig. 2 below). The situation changes radically when we pass to multiply charged ions of noble gases in the field of an X-ray laser (see Table 2): the ionization rate remains accessible for observation for $\gamma \geq 20$. Coulomb corrections increase in absolute value mainly due to an increase in the effective principal quantum number $n^{*}$ (this effect can be traced even in Table 1). As a result, Coulomb corrections increase the ionization probability by 5-7 orders of magnitude or more (see Fig. 3 below). The deviation of the Coulomb correction from the tunnel limit is also significant.

\footnotetext{
${ }^{9}$ Parameter $\gamma$ increases upon a decrease in intensity, but the ionization probability simultaneously becomes so low that its analysis is meaningless. It can be seen from Table 1 that in the case of helium, this is observed even for intensity $\mathscr{E}=10^{13} \mathrm{~W} / \mathrm{cm}^{2}$.
}

To estimate the accuracy of expression (25), we compared the ionization rate calculated using formula (28) with the results of numerical calculations [32]. Figure 1 shows the logarithms of the ionization rate for a hydrogen atom in the $1 s$ state in field (11) of the second harmonic of a titanium-sapphire laser with a photon energy of $\hbar \omega=3.10 \mathrm{eV}$ and in the field with a slightly lower frequency $(\hbar \omega=2.94 \mathrm{eV})$. Numerical results were obtained by two different methods, briefly described in Appendix C. The ionization rate calculated by the complex quasi-energy (Floquet) method is shown by dark circles. Triangles correspond to the results obtained from numerical solution of the timedependent Schrödinger equation in the field of a short pulse with a duration of ten field periods. The obvious perfect matching of the results of two independent numerical calculations in Fig. 1 point to the reliability of this method.

For not very high values of the adiabaticity parameter, the difference of Coulomb correction $Q_{C}$ from $Q_{0}$ is insignificant, which is demonstrated by the curves in Fig. 2 plotted for ionization of the hydrogen atom by the radiation field of a $\mathrm{Nd}$ laser with a wavelength of $1064 \mathrm{~nm}$. Here, the results of numerical calculation of 


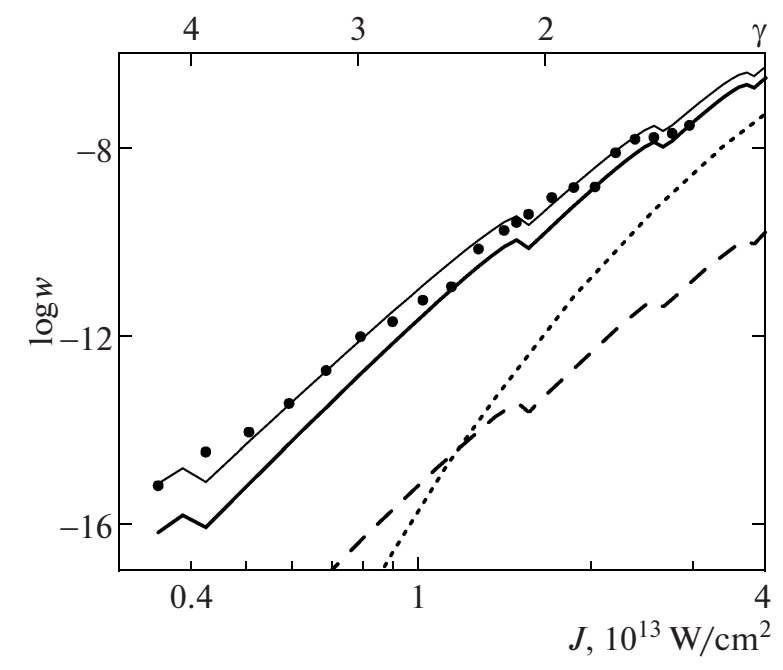

Fig. 2. Same as in Fig. 1 for a Nd laser with a wavelength of $1064 \mathrm{~nm}$. The thin solid line shows the ionization rate calculated taking into account correction $Q_{0}$ alone.

the ionization rate by the Floquet method are even closer to the analytical curve plotted by taking into account only correction $Q_{0}$ as compared to the curve calculated using formula (28).

Figure 3 shows the ionization rate of the $4 p_{0}$ state of the $\mathrm{Xe}^{17+}$ ion calculated using formulas (25), (27), and (28) and determined by numerical integration of the one-particle time-dependent Schrödinger equation for an electron in the effective potential in the electric field of a wave (see Appendix C). Comparison of the analytic and numerical results shows good quantitative agreement even in the case of xenon, although parameter $\mu=0.34$ is not very small in this case, and the bound state is characterized by a unit angular momentum, while analytic results were obtained for the $s$ states. Deviations of analytic results from numerically determined ionization rates are mainly associated with resonances disregarded in the given theory. The fact that it is resonances that are responsible for the discrepancy between numerical results and the theory is confirmed by comparison of Figs. 1a and $1 \mathrm{~b}$. In the former case, four-photon resonance with one of the Rydberg levels with the position controlled by the intensity due to dynamic Stark shift is observed in the range of intensities $(2-3) \times 10^{13} \mathrm{~W} / \mathrm{cm}^{2}$. In Fig. $1 \mathrm{~b}$, the wavelength that does not correspond to any specific laser is chosen so that there is no resonance. The coincidence of analytic and numerical results is almost perfect.

Apart from the numerical results considered above, we are aware of only one example of calculations of the multiphoton ionization probability of positive ions in the radiation field of a high-intensity X-ray laser, performed in [39] for ionization of xenon by the field with a photon energy of $\hbar \omega=12.7 \mathrm{eV}$ and an intensity of

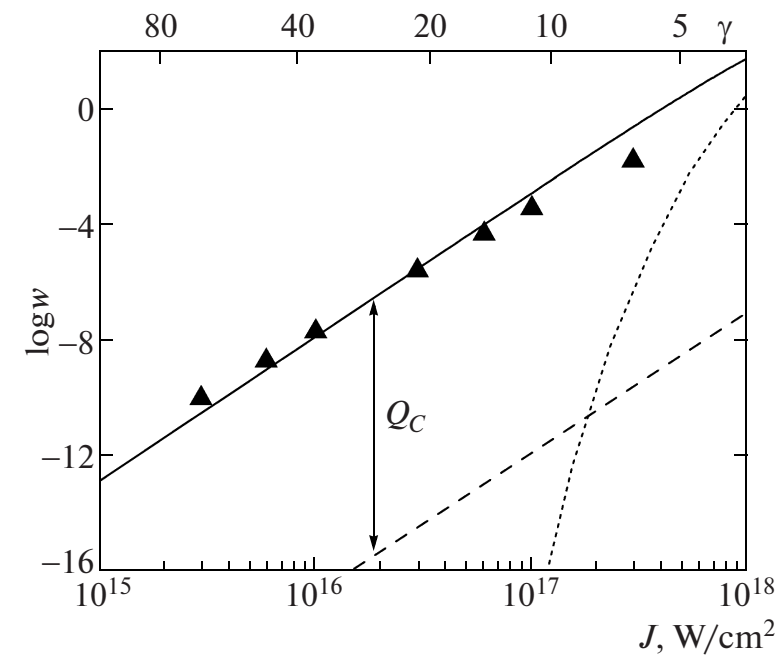

Fig. 3. Same as in Figs. 1 and 2 for the $\mathrm{Xe}^{17+}$ ion $(I=$ $434 \mathrm{eV}, 4 p_{0}$ shell) in the field of a X-ray laser with wavelength $\lambda=13.3 \mathrm{~nm}(\hbar \omega \approx 93 \mathrm{eV})$ [4]. Parameters $K_{0}, n^{*}$, and $\mu$ are $4.67,3.19$, and 0.34 , respectively; Coulomb correction $Q_{C} \approx 9 \times 10^{8}$.

$10^{13} \mathrm{~W} / \mathrm{cm}^{2}$ (i.e., under the conditions of experiment [7], in which charge states were observed up to $\mathrm{Xe}^{6+}$ inclusive). In [39], multiphoton cross sections

$$
\sigma_{N_{\min }}=w_{N_{\min }} / j^{N_{\min }}
$$

were determined numerically, where $N_{\min }$ is the minimal number of photons required for ionization (introduced above) and $j=c \mathscr{E}_{0}^{2} / 8 \pi \omega$ is the photon flux density. In experimental conditions [7], the flux density was $j \approx 5 \times 10^{30} \mathrm{~cm}^{-2} \mathrm{~s}^{-1}$. Knowing the cross sections, we can easily calculate the corresponding probabilities and compare them with analogous values calculated using formula (31). Comparison of our results with the probabilities calculated from the results of [39] demonstrate satisfactory agreement: formula (31) gives $w_{4}\left(\mathrm{Xe}^{3+}\right) \approx 4.2 \times 10^{-10}$ a.u. and $w_{5}\left(\mathrm{Xe}^{4+}\right) \approx 4.0 \times 10^{-13}$ a.u., while the corresponding values of these quantities determined in [39] are $w_{4}\left(\mathrm{Xe}^{3+}\right) \approx 2.7 \times 10^{-9}$ a.u. and $w_{5}\left(\mathrm{Xe}^{4+}\right) \approx 8.3 \times 10^{-12}$ a.u. The probabilities calculated from formula (31) are lower than the numerical results obtained in [39]. In our opinion, the systematic discrepancy is connected with the method of determining cross sections from these numerical calculations: the authors of [39] used the complex absorbing potential operating at distances $r>R_{0}=4$ a.u. from the nucleus. As a result, the electrons populating the upper-lying Rydberg levels were interpreted as ionized, which effectively increased the ionization cross section. As shown in recent experiment [40], the probability of exciting an electron to a Rydberg state during multiphoton ionization by the field of a high-intensity short pulse is not low at all. In the numerical calculations used here, electrons excited to Rydberg states are 
not treated as ionized (see Appendix C), while analytic results generally do not take into account the presence of the bound excited state.

\section{ELLIPTIC POLARIZATION}

\subsection{Monochromatic Field}

Let us now consider the correction to action (15) and the corresponding correction $Q_{0}$ to the ionization probability in the general case of elliptic polarization. The contribution to the function of action emerging due to the Coulomb interaction energy and regularized by matching to the asymptotic form of the atomic wavefunction has the form

$$
W_{C 0}=-i n^{*} \ln \left[\kappa r_{0}\left(\tau_{*}\right)\right]-i \frac{\mathscr{E}}{\omega} \int_{0}^{\tau_{*}} \frac{d \tau}{\sqrt{\mathbf{r}_{0}^{2}(\tau)}} .
$$

Here, $\tau_{*}$ is the matching point such that $\tau_{0}-\tau_{*} \ll \tau_{0}$ and $\kappa r_{0}\left(\tau_{*}\right) \gg 1[22,33]$. The subbarrier trajectory $\mathbf{r}_{0}(\tau)$ and the equation for the imaginary initial time $\tau_{0}$ correspond to $\mathscr{Z}=0$. Following [22], it is convenient to write the formula for the Coulomb correction in the form

$$
Q_{0}=\left\{\frac{2}{F} C(\gamma, \rho)\right\}^{2 n^{*}},
$$

where

$$
\begin{gathered}
C(\gamma, \rho)=\frac{\tau_{0}}{2 \gamma} \\
\times \exp \left\{\int_{0}^{\tau_{0}}\left(\frac{1}{\sqrt{\xi_{0}^{2}(\tau)}}-\frac{1}{\tau_{0}-\tau}\right) d \tau\right\}
\end{gathered}
$$

and $\xi_{0}=\omega \mathbf{r}_{0} / \kappa$. The latter expression was derived by regularizing formula (32) and does not contain arbitrary matching point $\tau_{*}$, which is convenient for numerical calculations. It can easily be shown that $\left|\xi_{0}(\tau)\right|=\tau_{0}-\tau+\ldots$ for $\tau \longrightarrow \tau_{0}$; consequently, singularities of the integrand in formula (33') are cancelled out and the integral always converges. It should be noted that expressions (32), (33), and (33') are valid not only for an arbitrary polarization, but also for an arbitrary (not necessarily monochromatic) pulse.

For a monochromatic elliptically polarized field

$$
\mathscr{E}(t)=\mathscr{E}_{0}\left(\mathbf{e}_{x} \cos \omega t+\rho \mathbf{e}_{y} \sin \omega t\right)
$$

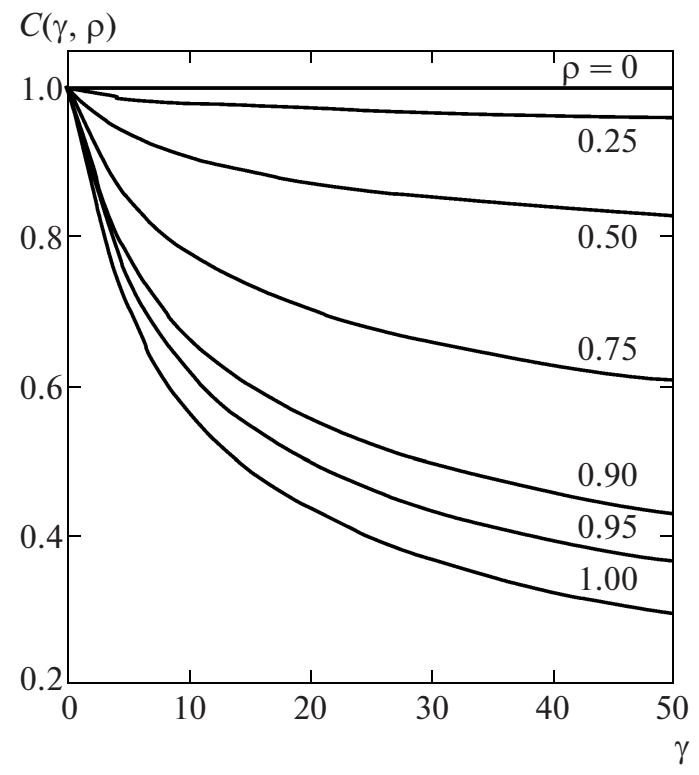

Fig. 4. Factor $C(\gamma, \rho)$ in Coulomb correction (33) as function of $\gamma$ for various values of ellipticity $\rho$.

trajectory $\mathbf{r}_{0}$ can be found analytically:

$$
\begin{gathered}
\mathbf{r}_{0}(\tau)=\frac{\mathscr{E}_{0}}{\omega^{2}}\left\{\left(\cosh \tau_{0}-\cosh \tau\right) \mathbf{e}_{x}\right. \\
\left.\quad+i \rho\left(\frac{\tau}{\tau_{0}} \sinh \tau_{0}-\sinh \tau\right) \mathbf{e}_{y}\right\}
\end{gathered}
$$

$$
\sinh ^{2} \tau_{0}\left[1-\rho^{2}\left(\operatorname{coth} \tau_{0}-\frac{1}{\tau_{0}}\right)^{2}\right]=\gamma^{2}
$$

Numerical calculations based on Eqs. (33'), (35), and (36) shows that (Fig. 4) factor $C(\gamma, \rho)$ decreases with increasing $\gamma$, the increase being especially strong for a nearly circular polarization. Substituting Eq. (35) into (33) and (33'), we arrive at the expression

$$
\begin{gathered}
Q_{0}=\exp \left\{-2 \operatorname{Im} W_{C 0}\right\} \\
=\exp \left\{2 n^{*}\left[\ln \left(2 K_{0} \tau_{0}\right)+J(\gamma, \rho)\right]\right\},
\end{gathered}
$$

where

$$
\begin{gathered}
J=\int_{0}^{1} d s\left\{\gamma \left[\left(\cosh \tau_{0}-\cosh \left[\tau_{0}(1-s)\right]\right)^{2}\right.\right. \\
\left.\left.-\rho^{2}\left((1-s) \sinh \tau_{0}-\sinh \left[\tau_{0}(1-s)\right]\right)^{2}\right]^{-1 / 2}-\frac{1}{s}\right\},
\end{gathered}
$$

$s=1-\tau / \tau_{0}$. For $\rho=0$, the integral can be evaluated analytically, $J=\ln \left(2 \gamma / \tau_{0}\right)$, which gives familiar expression (1) for the Coulomb correction in a linearly polarized field. 


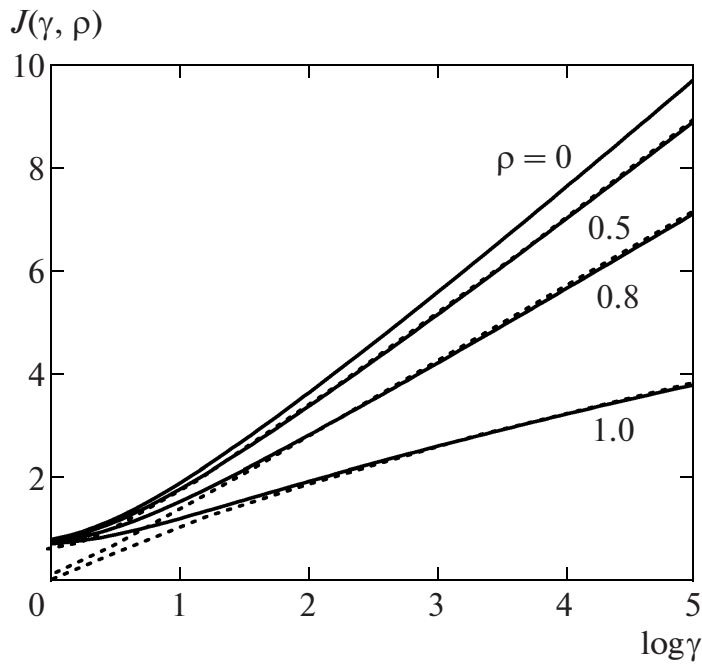

Fig. 5. Plots of functions (38) (solid curves) and (41) (dotted curves) of angle $\gamma$ for various values of ellipticity $\rho$. For $\rho=0.5$, the curves are practically indistinguishable in the entire range of parameter $\gamma$; for lower values of ellipticity, the difference between the exact result and the curve depicting formula (41) is even smaller.

In the multiphoton limit $\tau_{0} \gg 1$, we have the asymptotic form

$$
\begin{gathered}
\tau_{0}(\gamma, \rho) \\
=\left\{\begin{array}{l}
\ln \left(2 \gamma / \sqrt{1-\rho^{2}}\right), \quad 1-\rho^{2} \gg 1 / \ln (2 \gamma), \\
\ln (\gamma \sqrt{2 \ln \gamma}), \quad \rho^{2}=1
\end{array}\right.
\end{gathered}
$$

(asymptotic form $\tau_{0}$ is more complicated in a narrow transition region in the vicinity of $\rho= \pm 1$ ). In this case, expression (38) is simplified:

$$
\begin{aligned}
J \approx \int_{0}^{1} d s\left\{[ \tau _ { 0 } ^ { 2 } - \rho ^ { 2 } ( \tau _ { 0 } - 1 ) ^ { 2 } ] ^ { 1 / 2 } \left[\left(1-\exp \left(-\tau_{0} s\right)\right)^{2}\right.\right. \\
\left.\left.\quad-\rho^{2}\left(1-s-\exp \left(-\tau_{0} s\right)\right)^{2}\right]^{-1 / 2}-\frac{1}{s}\right\} .
\end{aligned}
$$

For $\gamma \gg 1$, we can write the following simple interpolation formula for integral (40):

$$
\begin{gathered}
J(\gamma, \rho)=\sqrt{\tau_{0}^{2}-\rho^{2}\left(\tau_{0}-1\right)^{2}} \frac{\arcsin \rho}{\rho} \\
-\frac{\pi \rho^{2}}{2\left[\tau_{0}\left(1-\rho^{2}\right)+\rho^{2}\right]}-\ln \tau_{0} .
\end{gathered}
$$

The curves plotted in Fig. 5 indicate that this formula for $\gamma \geq 10$ ensures high accuracy for all values of $\rho$; for a polarization far from circular $(\rho \leq 0.5)$, expression (41) matches exact result (40) up to $\gamma \approx 1$.

In the case of circular polarization, we have

$$
J \approx \frac{\pi}{2} \sqrt{2 \tau_{0}}-\ln \tau_{0}, \quad \tau_{0} \approx \ln (\gamma \sqrt{2 \ln \gamma}),
$$

and Coulomb correction (37) assumes the form

$$
Q_{0} \approx\left(2 K_{0}\right)^{2 n^{*}} \exp \left(\pi n^{*} \sqrt{2 \ln \gamma}\right) .
$$

\subsection{Adiabatic Limit}

If $\gamma \ll 1$ (which is usually observed for optical and IR lasers), all functions in expressions (35) and (38) can be expanded into power series in $\tau, \tau_{0}$, and $\gamma^{2}$. After simple but cumbersome calculations, we obtain [41]

$$
C(\gamma, \rho)=1-\rho^{2}\left[\frac{1}{18} \gamma^{2}-\frac{1}{45}\left(1-\frac{25}{72} \rho^{2}\right) \gamma^{4}+\ldots\right],
$$

which matches the results in Fig. 4 for $\gamma<2$ with an error on the order of $1 \%$; consequently, in this range of parameters, we can use expansion (43) without evaluating integral (38). Expression (43) can be generalized to the relativistic case (i.e., for a level with a binding energy comparable to rest energy $m c^{2}$ ). In this case, the coefficient of $\gamma^{2}$ no longer vanishes for linear polarization [41].

\subsection{Plane Wave of Arbitrary Shape}

Above, we considered ionization by the field of monochromatic wave (11); however, formulas (33) and $\left(33^{\prime}\right)$ are also valid in a more general case. Let us suppose that

$$
\mathscr{E}(t)=\mathscr{E}_{0} \varphi(\theta), \quad-\infty<\theta \equiv \omega t<\infty,
$$

where function $\varphi(\theta)$ defines the shape of the pulse. To evaluate Coulomb factor $C(\gamma, \rho)$, we can substitute into Eq. $\left(33^{\prime}\right)$ the subbarrier trajectory $\xi_{0}(\tau)$ for $\mathscr{E}=0$, which can be determined from the equations given in Appendix D. For $\gamma \ll 1$, the ionization probability is controlled by the behavior of field $\mathscr{E}(\tau)$ in the vicinity of its maximum. Assuming that

$$
\varphi(\theta)=1-\frac{a_{2}}{2 !} \theta^{2}+\frac{a_{4}}{4 !} \theta^{4}+\ldots, \quad a_{2}>0,
$$

for $\theta \longrightarrow 0$, we obtain (see [42] for details)

$$
C(\gamma)=1+\frac{1}{216}\left(a_{4}-a_{2}^{2}\right) \gamma^{4}+O\left(\gamma^{6}\right) \text {. }
$$

It should be noted that this expansion begins with $\gamma^{4}$ (and not with $\gamma^{2}$ ), which, however, is a peculiarity of linear polarization (cf. formula (43)). As a rule, the coefficient of $\gamma^{4}$ is numerically small (see examples in Appendix D); consequently, the Coulomb correction in domain $\gamma \leq 1$ can be used in the same form (1) as in the case of a constant field.

\section{CONCLUSIONS}

The analytic formulas for Coulomb corrections have been derived by the method of perturbation theory in the Coulomb field. It would be interesting to consider limitations imposed on the application of this method. Correction $x_{1}(\tau)$ to the subbarrier trajectory can be calculated in perturbation theory; conse- 
quently, the Coulomb field must be weaker than the laser field on the majority of the subbarrier trajectory. For $\gamma \gg 1$, this condition can be reduced to the inequality

$$
\gamma \mu \ll 1
$$

which is rather stringent. ${ }^{10}$ Indeed, it can be seen from Tables 1 and 2 that parameter (19) for the ground states of atoms and for positive ions of noble gases varies in the limits $\mu \approx 0.05-0.2$. In accordance with inequality (47), this means that the upper bound of the adiabaticity parameter is $\gamma \approx 10$.

However, Figs. 1 and 2 show that the applicability of result (25) is extended to the range of much larger values of $\gamma$. To establish a more exact criterion of applicability, we must compare the Coulomb corrections to action (i.e., the exponents of each factor in relation (25)) with the action accumulated in the laser field. In the multiphoton mode, this action is $K_{0}[\ln (2 \gamma)-1 / 2]$. As a result, we obtain a less stringent inequality instead of (47),

$$
\mu \ll 1 \text {, }
$$

which does not contain $\gamma$, but suggests that $\gamma \gg 1$.

Thus, if the condition $K_{0} \gg 1$ is satisfied, formulas (25) and (28) are applicable for arbitrary values of adiabaticity parameter $\gamma$ and ensure a correct passage to the limit of a weak field, in which perturbation theory of the lowest order in $N_{\text {min }}$ is valid. These results generalize the familiar expressions [22] for the rate of multiphoton ionization of atoms and positive ions to the case of a strong field of an arbitrary frequency. The most natural range of application of the above theory corresponds to experiments on ionization of matter in the field of powerful X-ray lasers; such experiments are being actively performed at present.

It should be noted that the inclusion of the Coulomb interaction in the Keldysh theory was considered in early publications $[22,45]$, but this problem had not been solved completely. Nikishov and Ritus developed a special diagram technique (exponentiation method [45]) and derived the following relation in the case of circular polarization:

$$
Q_{C}(\mathscr{E}, \omega)=\frac{w\left(\mathscr{E}_{0}, \omega\right)}{w_{\mathrm{sr}}\left(\mathscr{E}_{0}, \omega\right)}=\left(F \sqrt{1+\gamma^{2}}\right)^{-2 n^{*}}
$$

which is in qualitative agreement with formula (42) for $\gamma \gg 1$. On the other hand, the result $w=w_{\mathrm{sr}} \exp \left(c \sqrt{K_{0}}\right)$ described in [45] for linear polarization with a constant $c \sim 1$ does not coincide with formula (25) and is strange in our opinion: this expression for $\omega \longrightarrow 0$ is not transformed into familiar formula (1) for the Coulomb correction for ionization by a constant field.

\footnotetext{
${ }^{10}$ It is well known, however, that semiclassical formulas are often "pulled" beyond the range of their formal applicability [43, 44].
}

\section{ACKNOWLEDGMENTS}

The authors are grateful to S.P. Goreslavskii, D.F. Zaretskii, B.M. Karnakov, M.V. Fedorov, and N.I. Shevtsov-Shilovskii for fruitful discussions and to S.G. Pozdnyakov for his help in numerical calculations.

This study was partly supported by the Russian Foundation for Basic Research (project nos. 05-0204016-NNIO_a, and 07-02-01116).

APPENDIX A

\section{Kapitza's Method in the Ionization Problem}

In the case of ionization of an atom by the field of a linearly polarized wave, the imaginary part of action assumes the minimal possible values for 1D trajectories lying on the straight line directed along the polarization of the wave. Let us consider such a $1 \mathrm{D}$ trajectory in the field $U_{C}=-e^{2 \mathscr{E} /} / r$ of the atomic core and electromagnetic wave (11), which satisfies the equation

$$
m \ddot{x}=-\frac{\mathscr{L} e^{2}}{x^{2}}+e \mathscr{E}_{0} \cos \omega t
$$

(in this appendix, we return to dimensional $\hbar, e$ and $m)$. In a rapidly oscillating field, we represent, in accordance with the Kapitza method [29, 30], the motion of an electron as a displacement along a smooth trajectory $X(t)$ with small oscillations at frequency $\omega$ about this trajectory:

$$
x(t)=X(t)+\frac{e \mathscr{E}_{0}}{m \omega^{2}}(1-\cos \omega t) .
$$

The condition for the applicability of the averaging method has the form $\omega T \gg 1$, where $T$ is the characteristic time of classical motion in the Coulomb field [30] after emergence from under the barrier,

$$
T=2 \pi b^{3 / 2} \sqrt{\frac{m}{\mathscr{L} e^{2}}}=\frac{2 \pi}{\omega} \sqrt{\frac{2 K_{0}}{n^{*}}} .
$$

Here, $b=\hbar \kappa / m \omega$ is barrier width (14) and parameters $K_{0}$ and $n^{*}$ are defined in expressions (1) and (4). Thus, the Kapitza method is applicable under the multiphoton ionization conditions:

$$
\omega T \approx \sqrt{\frac{2 K_{0}}{n^{*}}}=\mu^{-1 / 2} \gg 1,
$$

where dimensionless parameter $\mu$ is defined in (19). In this case, condition $\gamma \gg 1$ is also assumed to be satisfied, which ensures the smallness of the amplitude of oscillations as compared to spatial scale $b$ characterizing the averaged motion of the electron:

$$
\frac{e \mathscr{E}_{0}}{m \omega^{2} b}=\gamma^{-1} \ll 1
$$


Passing in (A.1) to dimensionless variables $\xi=x / b$ and $\theta=\omega t$ and averaging over period $2 \pi / \omega$, we obtain

$$
\begin{gathered}
\frac{d^{2} \xi}{d \theta^{2}}=-\frac{\mu}{2 \pi} \int_{0}^{2 \pi} \frac{d \theta}{\left[\xi-\gamma^{-1}(1-\cos \theta)\right]^{2}}=-\frac{\partial u}{\partial \xi}, \\
U=-\frac{\mu}{\sqrt{\xi^{2}-2 \xi / \gamma}} .
\end{gathered}
$$

The motion corresponding to smooth coordinate $\xi(\theta)$ is conservative with an energy integral

$$
\frac{1}{2}\left(\frac{d \xi}{d \theta}\right)^{2}+\vartheta(\xi)=E=\text { const. }
$$

We are interested in a solution corresponding to the ionization threshold (i.e., zero electron velocity at infinity, $E=0$ ); it is this solution that controls the extremal $[12,28]$ trajectory along which the ionization probability attains its maximal value. In this case, we have

$$
(d \xi / d \theta)^{2} \sqrt{\xi^{2}-2 \gamma^{-1} \xi}=2 \mu .
$$

For $\gamma \gg 1$ and $\xi \geq 1$, potential $U$ can be reduced to the Coulomb potential and relation (A.8) gives

$$
\xi=\left[\xi_{0}^{3 / 2}+\frac{3}{2} \sqrt{2 \mu}\left(\theta-\theta_{0}\right)\right]^{2 / 3} .
$$

Here, $\xi_{0}$ and $\theta_{0}=0$ are the dimensionless initial point and initial instant of motion in real time. Relation (A.9) leads to the average velocity (drift momentum) of the electron emerging from under the barrier (18):

$$
p_{\|}=m \dot{X}\left(\theta=\theta_{0}\right)=\kappa \sqrt{2 \mu / \xi_{0}} \approx \kappa \sqrt{2 \mu},
$$

since $\xi_{0} \approx 1$ (for $\mu=0$, we have $\xi_{0}=1$ ). Thus, the point of emergence from under the barrier is not the point at which the particle comes to a halt even in the 1D case in contrast to the conventional semiclassical approximation [20].

Passing to imaginary time $\tau=-i \theta$, we obtain the boundary condition for subbarrier trajectory $\xi(\tau)$ :

$$
\xi(d \xi / d \tau)^{2}=-2 \mu, \quad \tau=0,
$$

which shows that this trajectory is not real-valued any longer in the presence of the Coulomb interaction. Consequently, for $\mu \neq 0$, the initial instant $t_{s}$ of motion is also naturally shifted from the imaginary time axis.

For motion in imaginary time, the laser field is not oscillating and the Kapitza method is inapplicable. For $\gamma \mu \ll 1$ (see relation (47)), the Coulomb field is smaller than the laser field on the majority of the subbarrier trajectory, so that the correction to the trajectory can be found from Eq. (20), which assumes the following form in variables $\xi$ and $\tau$ :

$$
\begin{gathered}
\frac{d^{2} \xi_{1}}{d \tau^{2}}=\frac{\mu}{\xi_{0}^{2}}, \\
\xi_{0}(\tau)=\frac{1}{\gamma}\left(\cosh \tau_{0}-\cosh \tau\right) \approx 1-\exp \left(\tau-\tau_{0}\right) .
\end{gathered}
$$

This equality was obtained taking into account the fact that the value of $\tau_{0} \approx \ln (2 \gamma) \gg 1$ for $\gamma \gg 1$ so that $\cosh \tau_{0} \approx \sinh \tau_{0}=\gamma$. Integrating relation (A.12) twice under initial conditions $\xi(\tau=0)=\xi_{0} \equiv 1+a$, $d \xi / d \tau(\tau=0)=i \sqrt{2 \mu}$, we obtain correction (21) to the trajectory. Real constant $a$ vanishes for $\mu=0$ and does not affect the ionization probability (see Appendix $B$ ).

\section{APPENDIX B}

\section{Calculation of the Correction to Action}

We will describe here the derivation of formula (23). Let us suppose that

$$
\mathbf{r}(t)=\mathbf{r}_{0}(t)+\mathbf{r}_{1}(t)+\ldots,
$$

where $\mathbf{r}_{0}$ is trajectory (10) disregarding the Coulomb interaction and $\mathbf{r}_{1}$ is the first-order correction in $\mathscr{E}$ (or in $\mu$ ) to this trajectory; here, $\mathbf{v}_{i}=\dot{\mathbf{r}}_{i} \equiv d \mathbf{r}_{i} / d t$.

To calculate correction (16), we note that the first term in the integrand can be evaluated by parts:

$$
\int_{t_{s}}^{\infty} \mathbf{v}_{0} \cdot \mathbf{v}_{1} d t=\left.\mathbf{v}_{0} \cdot \mathbf{r}_{1}\right|_{t=t_{s}} ^{t \rightarrow \infty}-\int_{t_{s}}^{\infty} \dot{\mathbf{v}}_{0} \cdot \mathbf{r}_{1} d t .
$$

As a result, the integrated term in formula (B.2) cancels out with the same term in function $F_{1}$ having the opposite sign. Since $\dot{\mathbf{v}}_{0}=-\mathscr{E}(t)$, the integral on the right-hand side of formula (B.2) also cancels out with the third term in formula (16). Analogous cancellations take place in derivation of the formula

$$
\operatorname{Im} W=\frac{1}{2} \int_{0}^{t_{s}}\left[\kappa^{2}+\mathbf{p}^{2}(t)\right] d t,
$$

which is valid [28] in the case of short-range potentials. The ionization probability is affected by only the imaginary part of action; consequently, the contribution from the upper limit in function $F_{1}$ is insignificant. For the same reason, it is sufficient to evaluate the integral in relation (16) only with respect to imaginary time $\omega t=i \tau, 0 \leq \tau \leq \tau_{0}, \omega t_{s}=i \tau_{0}$. Thus, the imaginary part of the function of action (16) is

$$
\begin{aligned}
\operatorname{Im} W_{C 1} & =-\frac{1}{2 \omega} \int_{0}^{\tau_{0}} \mathbf{v}_{1}^{2} d \tau+\left.\operatorname{Im}\left(\mathbf{r}_{0} \cdot \mathbf{v}_{1}+\mathbf{r}_{1} \cdot \mathbf{v}_{1}\right)\right|_{\tau=\tau_{0}} \\
& =-\frac{1}{2 \omega} \int_{0}^{\tau_{0}} \dot{x}_{1}^{2} d \tau+\left.\operatorname{Im}\left(x_{0} x_{1}+x_{1} \dot{x}_{1}\right)\right|_{\tau=\tau_{0}}
\end{aligned}
$$

(the trajectory is one-dimensional). Retaining contributions on an order no higher than the first in parameter $\mu$, we obtain from relation (21)

$$
\begin{gathered}
\dot{x}_{1}^{2}=2 \kappa^{2} \mu, \quad \operatorname{Im} x_{1}\left(\tau_{0}\right)=b \sqrt{2 \mu} \tau_{0}, \\
\operatorname{Re} \dot{x}\left(\tau_{0}\right)=\kappa \sqrt{2 \mu} .
\end{gathered}
$$


Finally, from relation (12), we have $x_{0}\left(\tau_{0}\right)=0$. As a result, we obtain from (B.4)

$$
\operatorname{Im} W_{C 1}=-\frac{\kappa^{2} \mu}{\omega} \tau_{0}+b \sqrt{2 \mu} \tau_{0} \kappa \sqrt{2 \mu},
$$

which leads precisely to (22). The real part of correction (16) is infinitely large at the lower integration limit, but this does not affect the ionization probability. In particular, constant $a$ in the Coulomb correction to trajectory (21) does not affect this probability.

\section{APPENDIX C}

\section{Numerical Methods}

The results of numerical calculations of the ionization rate presented in Figs. 1-3 were obtained using two different methods. The Floquet method (also known as the complex quasi-energy method) is based on representation of the solution to the Schrödinger equation for an atomic electron in the presence of a monochromatic electromagnetic wave in the form of a Fourier series with coefficients (position functions) that can be determined from the solution of an infinitely large system of ordinary differential equations. The eigenvalues of this system are complex and known as quasi-energies [46, 47]. The doubled imaginary part of the quasi-energy with the minus sign is the ionization probability for an atomic level per unit time. A detailed description of the Floquet method applied to the problem of photoionization of atoms in a strong field is given, for example, in [48]. The same publication also contains an easily accessible STRELO numerical code for computing the quasi-energies of atomic levels in a monochromatic field of an arbitrary polarization, which was used for obtaining our results represented in Figs. 1 and 2. For the hydrogen atom, the Floquet method is quite effective since the time for calculating quasi-energy for a given state and fixed parameters of the field (i.e., a single point in Figs. 1 and 2) using a medium-power multiprocessor computer is about $10 \mathrm{~s}$. In a purely monochromatic field, multiphoton resonances between bound states with energies depending on the applied field due to the dynamic Stark shift play a significant role (see, for example, Fig. 1a). With increasing field, the probabilities of excitation of multiphoton resonances also increases, which considerably complicates computations. For this reason, we failed to calculate the linewidths for the hydrogen atom using the Floquet method for intensities exceeding $10^{14} \mathrm{~W} / \mathrm{cm}^{2}$.

An alternative approach to calculating the ionization rate is based on direct numerical integration of the Schrödinger equation for an atomic electron in the field of a finite-duration laser pulse. From the analytic point of view, this approach boils down to solving the Cauchy problem with the initial condition corresponding to an electron in a certain bound state. The results shown in Figs. 1 and 3 by triangles were obtained using the Qprop code [49] for numerical integration of the time-dependent one-electron Schrödinger equation in a given effective atomic potential in the field of an electromagnetic wave with a time envelope corresponding to a pulse with a length of ten periods:

$$
\begin{gathered}
\mathscr{E}(t)=\mathscr{E}_{0} f(\omega t) \sin \omega t, \\
f(\theta)= \begin{cases}\theta / 4 \pi, & 0 \leq \theta<4 \pi, \\
1, & 4 \pi \leq \theta \leq 16 \pi, \\
(20 \pi-\theta) / 4 \pi, & 16 \pi<\theta \leq 20 \pi .\end{cases}
\end{gathered}
$$

To evaluate the ionization probability, norm $\mathcal{N}(t)$ (integral of the probability density over the volume) was determined in a sphere of large radius $R$ after termination of the laser pulse. Probability $1-\mathcal{N}(\infty)$ divided by pulse duration $\tau$ gives the value of the ionization rate. The same value can be estimated by evaluating the time derivative of $\mathcal{N}(t)$. It is important to note that choosing a large radius of the sphere $(R=$ 40 a.u. for the hydrogen atom), we exclude the interpretation of electrons excited to the Rydberg levels as ionized electrons. The results of calculations show that the fraction of such excited electrons is significant.

As compared to the Floquet method, the solution of the time-dependent Schrödinger equation is more convenient for a generalization to the case of a nonhydrogen-like atom or ion. State $4 p_{0}$ of a valence electron in the $\mathrm{Xe}^{17+}$ ion, which plays the role of the initial condition of the time-dependent Schrödinger equation, was constructed as an eigenstate in the effective potential

$$
U(r)=-\mathscr{L} / r-(54-\mathscr{L}) e^{-\beta r} / r,
$$

where $\mathscr{L}=18$, and parameter $\beta=7.93$ was chosen so that the $4 p_{0}$ state possessed the known ionization potential $I=434 \mathrm{eV}$.

Evaluation of the ionization rate for an energy level by solving the time-dependent Schrödinger equation requires a much longer time: the calculation of one point (triangle) in Figs. 1 and 2 on a multiprocessor computer takes several hours.

\section{APPENDIX D}

\section{Coulomb Factor C $(\gamma)$ for a Arbitrary-Shape Pulse}

In the case of a linearly polarized electromagnetic pulse of arbitrary shape (44), the subbarrier trajectory $\xi_{0}(\tau)$ for $\mathscr{E}=0$ (i.e., disregarding the Coulomb interaction) is determined from the equations

$$
\begin{gathered}
\ddot{\xi}_{0}=-\gamma^{-1} \varphi(i \tau), \\
\xi_{0}\left(\tau_{0}\right)=\dot{\xi}_{0}(0)=0, \quad \dot{\xi}_{0}^{2}\left(\tau_{0}\right)=1 .
\end{gathered}
$$

Here, $\xi_{0}=\omega \kappa^{-1} x_{0}(t), 0 \leq \tau \leq \tau_{0}, \varphi(\theta)$ defines the shape of the pulse, and the derivative with respect to imaginary time $\tau=-i \theta$ is denoted by dot. The last condition in (D.1) defines the "initial instant" $\tau_{0}$ of the subbarrier motion. 
In some cases, these equations can be solved exactly.

(a) For a monochromatic field $\varphi(\theta)=\cos \theta$, we obtain

$$
\xi_{0}(\tau)=\frac{1}{\gamma}\left(\cosh \tau_{0}-\cosh \tau\right), \quad \tau_{0}=\arcsin \gamma
$$

and the integral in Eq. (33') is evaluated analytically and gives $C(\gamma)=1$ for all values of $\gamma$ so that $Q_{1}=$ $(2 / F)^{2 n^{*}}$ is independent of $\gamma$ (i.e., on frequency $\omega$ for a fixed amplitude $\mathscr{E}_{0}$ ). The latter, however, is a specific feature of linear polarization and of the given shape of the field, which will be demonstrated in the following examples.

(b) For $\varphi(\theta)=1 / \cosh ^{2} \theta$ (soliton-like pulse), we have

$$
\begin{gathered}
\xi_{0}(\tau)=\frac{1}{\gamma} \ln \frac{\cos \tau}{\cos \tau_{0}}, \quad \tau_{0}=\arctan \gamma \\
C(\gamma)=1+\frac{1}{54} \gamma^{4}+O\left(\gamma^{6}\right) .
\end{gathered}
$$

(c) For a pulsed field $\varphi(\theta)=\left(1+\theta^{2}\right)^{-3 / 2}$, we obtain

$$
\begin{gathered}
\xi_{0}(\tau)=\frac{1}{\gamma}\left(\sqrt{1-\tau^{2}}-\sqrt{1-\tau_{0}^{2}}\right), \\
\tau_{0}=\frac{\gamma}{\sqrt{1+\gamma^{2}}}, \\
C(\gamma)=1+\frac{1}{6} \gamma^{4}-\frac{2}{15} \gamma^{6}+\ldots
\end{gathered}
$$

(d) In the above examples, coefficient $c_{4}=a_{4}-a_{2}^{2}$ in expansion (46) is nonnegative, as well as for $\varphi(\theta)=$ $\exp \left(-\theta^{2}\right), 1 / \cosh \theta$, etc. However, $c_{4}$ can also be smaller than zero in some cases. Let us suppose, for example, that

$$
\begin{gathered}
\varphi(\theta)=\exp \left\{-\frac{1}{2}(1-s) \theta^{2}\right\} \cos (\sqrt{s} \theta) \\
=1-\frac{1}{2} \theta^{2}+\frac{1}{24}\left(3-2 s^{2}\right) \theta^{4}+\ldots,
\end{gathered}
$$

where $-\infty<s \leq 1$. Then, in accordance with relation (46), we have

$$
C(\gamma)=1+\frac{1}{108}\left(1-s^{2}\right) \gamma^{4}+O\left(\gamma^{6}\right)
$$

and $c_{4}<0$ for $s<-1$. Thus, the Coulomb factor $C(\gamma)$ may either increase or decrease with increasing $\gamma$ depending on the shape of the pulse. It should be noted that for $0<s \leq 1$, Eq. (D.7) corresponds to a modulated pulse with a Gaussian envelope (this model is often used in laser physics); for $s \leq 0$, oscillations vanish and formula (D.7) describes a single pulse.

In experiments, ultrashort pulses are used [14] with a duration of only a few field periods and with a shape far from an ideal sinusoid. In this connection, the functions determining the dependence of the ioniza- tion rate on the Keldysh parameter and the momentum spectra of photoelectrons $[50,51]$ were calculated for various fields of type (44).

\section{REFERENCES}

1. J. Andruszkow, B. Aunte, V. Ayvazyan, N. Baboi, R. Bakker, V. Balakin, D. Barni, A. Bazhan, M. Bernard, A. Bosotti, J. C. Bourdon, W. Brefeld, R. Brinkmann, S. Buhler, J.-P. Carneiro, M. Castellano, P. Castro, L. Catani, S. Chel, Y. Cho, S. Choroba, E. R. Colby, W. Decking, P. Den Hartog, M. Desmons, M. Dohlus, D. Edwards, H. T. Edwards, B. Faatz, J. Feldhaus, M. Ferrario, M. J. Fitch, K. Flöttmann, M. Fouaidy, A. Gamp, T. Garvey, C. Gerth, M. Geitz, E. Gluskin, V. Gretchko, U. Hahn, W. H. Hartung, D. Hubert, M. Hüning, R. Ischebek, M. Jablonka, J. M. Joly, M. Juillard, T. Junquera, P. Jurkiewicz, A. Kabel, J. Kahl, H. Kaiser, T. Kamps, V. V. Katelev, J. L. Kirchgessner, M. Körfer, L. Kravchuk, G. Kreps, J. Krzywinski, T. Lokajczyk, R. Lange, B. Leblond, M. Leenen, J. Lesrel, M. Liepe, A. Liero, T. Limberg, R. Lorenz, Lu Hui Hua, Lu Fu Hai, C. Magne, M. Maslov, G. Materlik, A. Matheisen, J. Menzel, P. Michelato, W.-D. Möller, A. Mosnier, U.-C. Müller, O. Napoly, A. Novokhatski, M. Omeich, H. S. Padamsee, C. Pagani, F. Peters, B. Petersen, P. Pierini, J. Pflüger, P. Piot, B. Phung Ngoc, L. Plucinski, D. Proch, K. Rehlich, S. Reiche, D. Reschke, I. Reyzl, J. Rosenzweig, J. Rossbach, S. Roth, E. L. Saldin, W. Sandner, Z. Sanok, H. Schlarb, G. Schmidt, P. Schmüser, J. R. Schneider, E. A. Schneidmiller, H.-J. Schreiber, S. Schreiber, P. Schütt, J. Sekutowicz, L. Serafini, D. Sertore, S. Setzer, S. Simrock, B. Sonntag, B. Sparr, F. Stephan, V. A. Sytchev, S. Tazzari, F. Tazzioli, M. Tigner, M. Timm, M. Tonutti, E. Trakhtenberg, R. Treusch, D. Trines, V. Verzilov, T. Vielitz, V. Vogel, G. V. Walter, R. Wanzenberg, T. Weiland, H. Weise, J. Weisend, M. Wendt, M. Werner, M. M. White, I. Will, S. Wolff, M. V. Yurkov, K. Zapfe, P. Zhogolev, and F. Zhou, Phys. Rev. Lett. 85, 3825 (2000).

2. http://flash.desy.de

3. V. Ayvazyan, N. Baboi, I. Bohnet, R. Brinkmann, M. Castellano, P. Castro, L. Catani, S. Choroba, A. Cianchi, M. Dohlus, H. T. Edwards, B. Faatz, A. A. Fateev, J. Feldhaus, K. Flöttmann, A. Gamp, T. Garvey, H. Genz, Ch. Gerth, V. Gretchko, B. Grigoryan, U. Hahn, C. Hessler, K. Honkavaara, M. Hüning, R. Ischebeck, M. Jablonka, T. Kamps, M. Körfer, M. Krassilnikov, J. Krzywinski, M. Liepe, A. Liero, T. Limberg, H. Loos, M. Luong, C. Magne, J. Menzel, P. Michelato, M. Minty, U.-C. Müller, D. Nölle, A. Novokhatski, C. Pagani, F. Peters, J. Pflüger, P. Piot, L. Plucinski, K. Rehlich, I. Reyzl, A. Richter, J. Rossbach, E. L. Saldin, W. Sandner, H. Schlarb, G. Schmidt, P. Schmüser, J. R. Schneider, E. A. Schneidmiller, H.-J. Schreiber, S. Schreiber, D. Sertore, S. Setzer, S. Simrock, R. Sobierajski, B. Sonntag, B. Steeg, F. Stephan, K. P. Sytchev, K. Tiedtke, M. Tonutti, R. Treusch, D. Trines, D. Türke, V. Verzilov, R. Wanzenberg, T. Weiland, H. Weise, M. Wendt, I. Will, S. Wolff, K. Wittenburg, M. V. Yurkov, and K. Zapfe, Phys. Rev. Lett. 88, 104802 (2002). 
4. A. A. Sorokin, S. V. Bobashev, T. Feigl, K. Tiedtke, H. Wabnitz, and M. Richter, Phys. Rev. Lett. 99, 213002 (2007).

5. H. Wabnitz, L. Bittner, A. R. B. de Castro, R. Döhrmann, P. Gürtler, T. Laarmann, W. Laasch, J. Schulz, A. Swiderski, K. von Haeften, T. Möller, B. Faatz, A. Fateev, J. Feldhaus, C. Gerth, U. Hahn, E. Saldin, E. Schneidmiller, K. Sytchev, K. Tiedtke, R. Treusch, and M. Yurkov, Nature (London) 420, 482 (2002).

6. T. Laarmann, A. R. B. de Castro, P. Gürtler, W. Laasch, J. Schulz, H. Wabnitz, and T. Möller, Phys. Rev. Lett. 92, 143401 (2004).

7. H. Wabnitz, A. R. B. de Castro, P. Gürtler, T. Laarmann, W. Laasch, J. Schulz, and T. Möller, Phys. Rev. Lett. 94, 023001 (2005).

8. N. Stojanovic, D. von der Linde, K. SokolowskiTinten, U. Zastrau, F. Perner, E. Förster, R. Sobierajski, R. Nietubyc, M. Jurek, D. Klinger, J. Pelka, J. Krzywinski, L. Juha, J. Cihelka, A. Velyhan, S. Koptyaev, V. Hájková, J. Chalupsky, J. Kuba, T. Tschentscher, S. Toleikis, S. Düsterer, and H. Redlin, Appl. Phys. Lett. 89, 241909 (2006).

9. S. W. Epp, J. R. Crespo López-Urrutia, G. Brenner, V. Mäckel, P. H. Mokler, R. Treusch, M. Kuhlmann, M. V. Yurkov, J. Feldhaus, J. R. Schneider, M. Wellhöfer, M. Martins, W. Wurth, and J. Ullrich, Phys. Rev. Lett. 98, 183001 (2007).

10. G. S. Voronov and N. B. Delone, Zh. Éksp. Teor. Fiz., Pis'ma Red. 1 (2), 42 (1965) [JETP Lett. 1 (2), 66 (1965)].

11. W. Becker, F. Grasbon, R. Kopold, D. B. Miloševič, G. G. Paulus, and H. Walther, Adv. At., Mol., Opt. Phys. 48, 35 (2002).

12. V. S. Popov, Usp. Fiz. Nauk 174 (9), 921 (2004) [Phys. - Usp. 47 (9), 855 (2004)].

13. A. Becker and F. H. M. Faisal, J. Phys. B: At., Mol. Opt. Phys. 38, R1 (2005).

14. D. B. Miloševič , G. G. Paulus, D. Bauer, and W. Becker, J. Phys. B: At., Mol. Opt. Phys. 39, R203 (2006).

15. L. V. Keldysh, Zh. Éksp. Teor. Fiz. 47, 1945 (1964) [Sov. Phys. JETP 20, 1307 (1964)].

16. F. H. M. Faisal, J. Phys. B: At. Mol. Phys. 6, L89 (1973); H. R. Reiss, Phys. Rev. A: At., Mol., Opt. Phys. 22, 1786 (1980).

17. A. I. Nikishov and V. I. Ritus, Zh. Éksp. Teor. Fiz. 50 (1), 255 (1966) [Sov. Phys. JETP 23 (1), 168 (1966)].

18. A. M. Perelomov, V. S. Popov, and M. V. Terent'ev, Zh. Éksp. Teor. Fiz. 50 (5), 1393 (1966) [Sov. Phys. JETP 23 (5), 924 (1966)]; Zh. Éksp. Teor. Fiz. 51 (1), 309 (1966) [Sov. Phys. JETP 24 (1), 207 (1966)].

19. N. L. Manakov, M. V. Frolov, B. Borca, and A. F. Starace, J. Phys. B: At., Mol. Opt. Phys. 36, R49 (2003).

20. L. D. Landau and E. M. Lifshitz, Course of Theoretical Physics, Vol. 3: Quantum Mechanics: Non-Relativistic Theory (Nauka, Moscow, 1989; Butterworth-Heinemann, Oxford, 1991).

21. Yu. N. Demkov and G. F. Drukarev, Zh. Éksp. Teor. Fiz. 47, 918 (1964) [Sov. Phys. JETP 20, 614 (1964)].
22. A. M. Perelomov and V. S. Popov, Zh. Éksp. Teor. Fiz. 52 (2), 514 (1967) [Sov. Phys. JETP 25 (2), 336 (1967)].

23. D. R. Hartree, Proc. Cambridge Philos. Soc. 24, 89 (1927).

24. S. L. Chin, C. Rolland, P. B. Corkum, and P. Kelly, Phys. Rev. Lett. 61, 153 (1998).

25. M. V. Ammosov, N. B. Delone, and V. P. Kră̆nov, Zh. Éksp. Teor. Fiz. 91 (6), 2008 (1986) [Sov. Phys. JETP 64 (6), 1191 (1986)].

26. V. S. Popov, Usp. Fiz. Nauk 169 (7), 819 (1999) [Phys._Usp. 42 (7), 733 (1999)].

27. V. S. Popov, V. P. Kuznetsov, and A. M. Perelomov, Zh. Éksp. Teor. Fiz. 53 (1), 331 (1967) [Sov. Phys. Usp. 26 (1), 222 (1967)].

28. V. S. Popov, Preprint No. 13-04, ITÉF (Institute of Theoretical and Experimental Physics, Moscow, 2000); Yad. Fiz. 68 (4), 717 (2005) [Phys. At. Nucl. 68 (4), 686 (2005)].

29. P. L. Kapitsa, Zh. Éksp. Teor. Fiz. 21, 588 (1951); Usp. Fiz. Nauk 44, 7 (1951).

30. L. D. Landau and E. M. Lifshitz, Course of Theoretical Physics, Vol. 1: Mechanics (Nauka, Moscow, 1989; Butterworth-Heinemann, Oxford, 1991).

31. S. V. Popruzhenko, G. G. Paulus, and D. Bauer, Phys. Rev. A: At., Mol., Opt. Phys. 77, 053409 (2008); S. V. Popruzhenko and D. Bauer, J. Mod. Opt. 55, 2573 (2008).

32. S. V. Popruzhenko, V. D. Mur, V. S. Popov, and D. Bauer, Phys. Rev. Lett. 101, 193003 (2008).

33. V. S. Popov, V. D. Mur, and S. V. Popruzhenko, Pis'ma Zh. Éksp. Teor. Fiz. 85 (5), 275 (2007) [JETP Lett. 85 (5), 223 (2007)]; S. V. Popruzhenko, V. D. Mur, and V. S. Popov, Phys. Lett. A 372, 59167 (2008).

34. H. Bateman and A. Erdelyi, Higher Transcendental Functions (McGraw-Hill, New York, 1955; Nauka, Moscow, 1966), Vol. 1.

35. Handbook of Mathematical Functions with Formulas, Graphs, and Mathematical Tables, Ed. by M. Abramowitz and I. A. Stegun (Dover, New York, 1972; Nauka, Moscow, 1979).

36. A. Becker, L. Plaja, P. Moreno, M. Nurhuda, and F. H. M. Faisal, Phys. Rev. A: At., Mol., Opt. Phys. 64, 023408 (2001).

37. I. Bersons, Phys. Lett. A 84, 364 (1981); I. Ya. Bersons, Zh. Éksp. Teor. Fiz. 83 (4), 1276 (1982) [Sov. Phys. JETP 56 (4), 731 (1982)].

38. H. A. Kramers, Philos. Mag. 46, 836 (1923).

39. Robin Santra and Chris H. Greene, Phys. Rev. A: At., Mol., Opt. Phys. 70, 053401 (2004).

40. T. Nubbemeyer, K. Gorling, A. Saenz, U. Eichmann, and W. Sandner, Phys. Rev. Lett. 101, 233001 (2008).

41. V. S. Popov, V. D. Mur, B. M. Karnakov, and S. G. Pozdnyakov, Phys. Lett. A 358, 21 (2006).

42. V. S. Popov, Doctoral Dissertation in Mathematical Physics (Institute of Theoretical and Experimental Physics, Moscow, 1974).

43. R. E. Langer, Phys. Rev. 75, 1573 (1949).

44. A. B. Migdal, Qualitative Methods in Quantum Theory (Nauka, Moscow, 1975; Benjamin, London, 1977). 
45. A. I. Nikishov and V. I. Ritus, Zh. Éksp. Teor. Fiz. 52 (1), 223 (1967) [Sov. Phys. JETP 25 (1), 145 (1967)].

46. Ya. B. Zel'dovich Zh. Éksp. Teor. Fiz. 51 (5), 1492 (1966) [Sov. Phys. JETP 24 (5), 1006 (1966)]; Usp. Fiz. Nauk 110, 139 (1973) [Sov. Phys._Usp. 16, 427 (1973)].

47. V. I. Ritus, Zh. Éksp. Teor. Fiz. 51 (5), 1544 (1966) [Sov. Phys. JETP 24 (5), 1041 (1966)].

48. R. M. Potvliege, Comput. Phys. Commun. 114, 42 (1998).
49. D. Bauer and P. Koval, Comput. Phys. Commun. 174, 396 (2006).

50. V. S. Popov, Laser Phys. 10, 1033 (2000); V. S. Popov, Pis'ma Zh. Éksp. Teor. Fiz. 73 (1), 3 (2001) [JETP Lett. 73 (1), 1 (2001)]; Zh. Éksp. Teor. Fiz. 120 (2), 315 (2001) [JETP 93 (2), 278 (2001)].

51. B. M. Karnakov, V. D. Mur, and V. S. Popov, Pis'ma Zh. Éksp. Teor. Fiz. 88 (7), 495 (2008) [JETP Lett. 88 (7), 423 (2008)].

Translated by N. Wadhwa 